\title{
Artificial Intelligence Price Emulator: A Study on Cryptocurrency
}

\author{
Apoorva Ganapathy ${ }^{*}$, Taposh Kumar Neogy ${ }^{2}$
}

${ }^{1}$ Senior Developer, Adobe Systems, San Jose, California, USA

${ }^{2}$ Assistant Professor (Accounting), Institute of Business Administration (IBA), National University, Rajshahi, BANGLADESH

*Corresponding Contact:

Email: apganapa@adobe.com

\begin{abstract}
The cryptocurrency Artificial intelligence price emulator is a software programmed to collect cryptocurrency market data, analyze the data and predict the market price using the collected data. Computer emulators are programmed to mimic and copy behaviors or other software/hardware. The reason for emulation is to get to a particular result as quickly as possible. Machine learning is the ability of computers to read and process data while learning from the data with human interference or influence. This work focused majorly on how cryptocurrency market prices can be emulated using Artificial Intelligence with machine learning abilities. It also looked into the advantages of using the software for crypto investors. Some of which is the reduced time of research, reduction of risk, among others.
\end{abstract}

Key Words: Cryptocurrency, Artificial Intelligence, Emulator, Machine Learning, Blockchain, Volatility, Coin, Algorithms

\section{INTRODUCTION}

Cryptocurrencies have become increasingly popular, especially on the internet, social media, and various news platforms. It is still very complex for most people as most people still find it strange to understand. They are also called digital currencies. Digital currencies were at a point utilized by just technical savvy people with digital networking skills. The popularity of the use of cryptocurrencies has dragged mainstream investors into cryptocurrency investment.

A cryptocurrency is a form of digital currency that can be held as an asset. Cryptography is deployed to make sure transactions are secured and anonymous. It also prevents counterfeiting and fake transaction hasher inventions. Fiat currencies are centralized, issued, and monitored by a central authority, usually the country's central bank. Cryptocurrencies are unregulated and decentralized. This means that there are no central government agencies issuing it. This is a point of attraction for major investors because its existence and transactions are not subject to government interference or influence. It is based on 
blockchain technology. Bitcoin and Ethereum remain the most popular cryptocurrencies, with also the most significant market capitalization. However, over 5,000 cryptocurrencies exist in the digital world today, and more are currently being created every day. Cryptocurrencies can be used just like regular Fiat currencies to purchase things, items, goods, and services online and in the real world (Vadlamudi, 2016). Most persons see cryptocurrencies are an investment like other property investments. I.e., just like investing in the stock market or investing in things like gold and other precious metal.

Cryptocurrency is still new and quite risky despite the excitement surrounding it. To understand it, there is a need to conduct thorough research. A more technical explanation of cryptocurrencies is that they are a network of data referred to as hash, encrypted to represent a currency unit. They are like data imputed in a systems database. They cannot be manipulated by any government. These data are subject to certain conditions before they may be altered. Mathematical algorithms are used to keep them secured.

In sending any cryptocurrency to somebody, a restricted message will be created and sent to the cryptocurrency's database. This message would also be signed with a unique security key. The cryptocurrency's network would mark and check the cryptocurrency to ensure that the same crypto has not been sent to another person before. Records of transactions are kept and monitored. After the check is concluded and confirmed that there is available cryptocurrency in your wallet, it will be transferred to the receiver, and they become his.

\section{Brief History of Cryptocurrencies}

The imagination of a digital currency may be seen as new, being a little less than 50 years old idea. Actual use of cryptocurrency may also be well below 15years. An understanding of the history and how cryptocurrency started may also help you understand what it is and what the future holds for it. Attempts to make a digital currency started as early as the 1980s. David Chaum, a cryptographer, created ecash. He tried to create an electronic money. Ecash transactions were done using Digicash. Digicash was a cryptographic form of payment that needed software and unique encrypted keys to the receiver. These processes and systems failed. Satoshi Nakamoto made another attempt. He founded bitcoin in 2008 by removing all the barriers in the Ecash and Digicash systems. It became the first digital currency to succeed. A network was used instead of the main server. Technically, Bitcoin involves sharing data using peer-to-peer sharing systems. This would mean that files are transferred or shared instead of downloading from a single server. Ecash used a server to avoid double-spending of a single asset (Ujwala et al., 2012). That is, a person uses particular crypto for multiple transactions. Most banks and financial organizations use a central server to avoid double-spending. The central server keeps records of transactions.

According to statists.com, there are over 5,000 digital coins, a major increase compared to the number available in 2013; according to statists.com. A large percentage of the available coin remain insignificant as it is easy to create a cryptocurrency. Most people believe that 90 percent of the cryptocurrency market is held by $1 \%$ of the cryptocurrencies.

\section{Terms to Know in Cryptocurrency}

There are several significant phrases and words to know to understand cryptocurrency.

\section{Block chain}

The name is coined from the arrangement of records. Single records are referred to as blocks. They are also connected together in a list known as chains. The Blockchain network 
is open to the public to read through. The public cannot alter them, and only the owners can update them. Most cryptocurrencies are based on blockchain. It is enabled on different computers on the internet through the peer-to-peer network. Blockchain keeps a digital record of all cryptocurrency transactions.

\section{Hash}

Hashing is used to secure data and prevent them from compromise and interference. They are codes of numbers and alphabets created using a mathematical algorithm. Hashes names of a specific file or type of cryptocurrency. There cannot be a single hash for more than one unit of cryptocurrency. Hashes cannot also be reversed engineered. They are also the security mechanism that prevents multiple spending if a single cryptocurrency unit.

\section{Peer to peer}

Cryptocurrency transactions involve multiple parties, the sender and the receiver. The P2P payment system usually enables the digital transfer of a cryptocurrency from one peer (party sending) to another peer (party receiving). P2P payments can be made using internetenabled devices or computers such as mobile phones, laptops, and so on.

\section{The public and private key}

Public keys are cryptographic keys that anyone can use for encryption. Persons or groups use private keys. This type of key is no open to the public for use as they are only known to the group members or the individuals. Most cryptocurrency transactions use keys. They aren't signature keys but means of ownership over wallets using mathematical algorithms.

\section{The Surging Popularity of Cryptocurrencies}

More persons are becoming aware of cryptocurrencies, and they are becoming more popular. The increased popularity may be related to the decentralized and secured nature of cryptocurrencies. It also has low fees compared to fiat. All the factors and other cryptocurrency features have played roles in attracting investors and traders. Sometimes using fiat for online transactions attracts high fees and rates. Cryptocurrency transactions involve low fees, and this attracts a lot of users. The decentralized nature of cryptocurrencies also plays its role. When a particular government or country faces economic or social disturbances, it would usually affect its currency. People avoid this disturbance by going into cryptocurrency investment where the government's influence is restricted, and the country's situation does not affect the stability of the investment. Cryptocurrency currencies have continued to increase in value. Bitcoin, for instance, rose from just below a thousand dollars in 2013 to over sixty thousand dollars in 2021. This has made it very attractive to investors who see it as a means to save wealth. The increased popularity can also be traced to the increase in understanding of cryptocurrencies. It has become less complex and simple to utilize. Companies like Binance, coinmarketcap, trustwallet, and so on have come up to make it easier to access cryptos. More people have now become aware of cryptocurrencies, and they've now understood that it's an alternative to Fiat currencies. In all, most questions concerning cryptocurrencies have been answered. It may not be up to the maximum level, but knowledge of crypto is spreading fast.

Cryptocurrency also makes transactions anonymous and, the identity of the parties is kept secret. Using cryptocurrency as a means of payment ensures identity protection compared to the traditional methods and platforms (Vadlamudi, 2015). This factor is also a point of attraction for investors who do not want to expose their identity. 
The promising future of cryptocurrency is the reason some investors are buying in. The growth of technology has been massive. We use one form of technology or the other every day (Vadlamudi, 2017). More persons are becoming technologically savvy and more inclined. Most investors see cryptocurrencies as the future money and have adopted them as part of the growing technological advancement. Cryptocurrency and the blockchain Platform has been held as new a technological development that is changing the world presently and would have a significant role in the world in time to come.

\section{Classes of Cryptocurrencies}

There are several classes of cryptocurrencies. However, the distinction between them all isn't huge or significant. The three main classes include:

- Bitcoin

- Altcoin

- Stablecoin

Bitcoin: It was created by Satoshi Nakamoto, who is seen as many as the father of cryptocurrencies. Obviously, Bitcoin isn't the first cryptocurrency to exist. It was built in 2008 and became popular in 2009. It is seen as the most successful cryptocurrency. Based on blockchain technology which allowed for a secured decentralized network that uses end-to-end encryption from transactions. The huge potential of Bitcoin was not known back then. Bitcoin can be shared using the P2P payment system. It is also becoming generally accepted by different firms, e.g., PayPal, Dell, Microsoft. Tesla has recently removed it from the list of currency accepted and replaced it with Dogecoin.

Altcoin: Altcoin is shortened from 'alternative coins.' They are an alternative to bitcoin. They sprung up following Bitcoin's success. Most developers have tried to make coins to follow in the footsteps of Bitcoin. Some solve particular problems associated with Bitcoin use. The majority of coins available are altcoins. Over four thousand altcoins exist. Major altcoins are Ethereum, litecoin, stellar, Dogecoin, BNB, Monero, Dash, Cardano, and so on. While bitcoin was designed as a form of currency, Ethereum was not designed as a digital currency. It was designed as a platform for creating blockchain applications.

Stablecoin: Stablecoin is created for asset stability. The cryptocurrency market is majorly unstable and highly volatile. Stablecoins were created as a means of storing value in the cryptocurrency world. As the prices of major cryptocurrencies fluctuate now and then, it becomes almost impossible to manage. Stablecoins are tethered to their reserve assets, and this brings about stability in price (Neogy \& Paruchuri, 2014). They bring the stability of price that comes with fiat and the anonymity feature that comes with cryptocurrency, like and hybrid currency. The value of Stablecoins is tethered to one currency or multiple currencies and placed in reserve to maintain value. Examples of Stablecoins are Tether (USDT) and Binance USD (BUSD).

\section{Some Other Popular Cryptocurrencies}

\section{Litecoin}

Litecoin is sometimes called the silver Bitcoin of the similarities it shares with Bitcoin. It was created in 2011 by Charlie Lee, a former Google engineer. It is also decentralized and uses block technology. It was one of the first follow up to Bitcoin. It concluded transactions faster than Bitcoin by blocks. 


\section{Ethereum}

They were launched in 2015 on a decentralized platform that prevents fraud and influence from third parties. Transactions on the Ethereum network are called ether. Ethereum split into Ethereum Classic and Ethereum due to some exploit. Unlike most cryptocurrencies, Ethereum doesn't run on the blockchain Platform.

\section{Ripple}

It was created in 2012. It came up as digital currency that doesn't need to be mined. It reduces the consumption of computer power and increases the transaction rate. It is also centralized. Its main purpose is to enable speedy transaction processes with low fees and charges. Major Banks have started using ripple technology and protocols.

\section{Dogecoin}

It started as a meme and a joke. The dogecoin community has increased remarkably. Over A hundred billion dogecoins were mined before early 2018. This is a remarkable performance being that the coin had was released about three years before then (Paruchuri, 2015). Dogecoins have been used for several humanitarian works. For example, it was used to generate funds to help send a Jamaican team to the Olympics in Sochi and help disabled children. In 2021 the value of Dogecoin rose remarkably to heights reaching close to $\$ 1$ mark. It is backed majorly by Elon Musk, Tesla CEO. Elon Musk, in May 2021, announced that Dogecoin would replace Bitcoin as a means of payment.

\section{Volatile Cryptocurrency Market}

In financial markets price of assets and stocks change in price from time to time. A price change can be positive, that is, when there is a steady price increase or decrease on a normal scale. It can also be a negative or sharp change. This occurs when the price change drastically upward or downwards. Positive volatility helps in different ways. It allows traders to profit by buying when the price is low and selling when the price is high. Negative volatility predicts asset value change difficult and almost impossible as the price changes too fast and could go to the extreme of both ways in a short time. The cryptocurrency market volatility is quite different from now foreign exchange and stock markets volatility. There is almost no index to quantify cryptocurrency market volatility. To know the market, one must have to look through the charts to see the trough peaks and how they go up and down the charts rapidly and in a short time. For instance, bitcoin rose by $2000 \%$ in 2017 and down back by $2000 \%$. It went up to $\$ 56000$ in 2021 before the drop back $\$ 30,000$. There are several reasons for cryptocurrency volatility. Mainly news, development, specular, and so on determine the market flow and fuels the prices. The effect of all this is quite overhyped. The cryptocurrency market is not as solid as that of the traditional investment market. Despite the high volatility of the cryptocurrency market, institutional investors have started to get into the cryptocurrency with more confidence.

Bitcoin volatility index just came up to track the volatility of the Bitcoin currency using the previous market cap. What influences crypto market volatility?

Negative news: Investors, traders, and users of cryptocurrencies are usually scared by news or statements from prominent persons or the government. For instance, fear of government restrictions in the past affected the market negatively, and tweets Elon Musk made the price of Dogecoin sour to its all-time high in 2021. News and statement have determined the price of cryptos vs. fiat up or down rapidly and in a short time. 
Uncertain future of cryptocurrency: Cryptocurrency volatility is majorly driven by public perception. Many users see it as a form of saving and storing wealth. This stored wealth may be used or transferred to someone else for one service or the other in the future. Cryptocurrency volatility currently makes it seem almost uncertain in terms of store value.

Big investors or Hodler: Hodler is a person who holds cryptocurrency in anticipation of the future rise in value. Big investors also determine the crypto market volatility. That is, people who have a huge amount or large percentage of the total circulating supply. When an investor with over $\$ 10 \mathrm{~m}$ worth of Bitcoin decides to liquidate or transfer within a short period, this type of transaction will affect the price of the cryptocurrency.

Security threats: When the cryptocurrency's ecosystem is faced with a security threat, maybe a possible hack, and the market volatility will be greatly affected. For instance, the Korean cryptocurrency Yapian Youbit went bankrupt after two hacks on its system.

Report of possible hacks on any cryptocurrency network would cause panic and substantial dumping of the cryptocurrency. This would cause the market to be volatile. In all, cryptocurrency market volatility is affected by multiple factors. The factors affect the price and cause the price movement up or down.

\section{Cryptocurrency Artificial Intelligence Price Emulator}

Artificial Intelligence, also called AI, is automated machines programmed to simulate and emulate human Intelligence and actions. Through protocols and script, they learn, think, and perform tasks just like humans do by mimicking and copying human actions. Different Industries have employed AI to carry out tasks and functions. This has led to the increased influence of AI across several industries including, health, agriculture, finance education, and so on (Paruchuri, 2017). Advanced development in AI technology is machine learning. A machine can automatically learn and study the input and output of data automatically without human assistance. AI programmed by scripts which set protocols and commands learn, reason and perceive things the way human do and take actions using algorithms that would result in the best results possible (Ganapathy, 2016). Everyone wants to go into cryptocurrency as we are in the crypto boom era. The cryptocurrency market is also still highly volatile, and prices fluctuate remarkably sharp upwards and downwards.

Cryptocurrency Artificial Intelligence price emulator is a tool that can be used to predict cryptocurrency market swings. It will use data from various streams and figure out for each vertical of cryptocurrency what the price would be after 12 months and further. The cryptocurrency price emulator will analyze the history, current market trend, cryptocurrency-related tweets, and sentiments behind them to reach a calculated and analyzed result of the future price. The cryptocurrency Artificial Intelligence price emulator would function using the machine learning concept. It will learn, identify, process, and input data without human intervention. Different persons may utilize the tool in different areas, such as prospective investors, traders, and holders of cryptocurrencies.

Predicting the cryptocurrency market price is the main goal of the emulator. The highly volatile cryptocurrency market price is mostly unpredictable. It follows a random sequence and Pattern that makes it difficult to determine. Cryptocurrency prices fluctuate at inconsistent rates. The cryptocurrency price emulating AI predicts change in using the news feeds. It predicts the news that could affect the price of cryptocurrency price instead of the crypto price itself. The AI gathers data and combines them for analysis and machine learning to create more knowledge about a particular cryptocurrency price in the future. 
Cryptocurrency price volatility is caused by some factors previously discussed-decisions of a big investor or many investors and human sentiment in trading. The sharp changes in cryptocurrency prices form a random price sequence. This can be seen when analyzing charts of specific range and noting the point of increase and decrease in value of crypto. This analysis usually would reveal that there is a sequence to price fluctuations and stability. The cryptocurrency price emulator uses these patterns to determine the future price. Users of the software would also find it useful and as a tool for income generation. The cryptocurrency price emulating AI can read thousands of data in minutes. It can also understand historical and reoccurring patterns that humans may not notice for predictive trading in cryptocurrency (Narayana et al., 2012). The cryptocurrency price emulator Analysis social media content, like posts and comments, write-ups, articles, and so on, about cryptocurrency. In traditional financial trade, sentiments and become quite popular and a term used frequently among traders.

\section{Why You should use Cryptocurrency Al, the Price Emulator}

Using a cryptocurrency price emulator to determine the future price and best investment can increase a crypto investor's ability to trade. It gives the trader a competitive advantage by reducing decision-making processes and giving important reports to enhance the best actions. Investors in traditional finance Markets use manual analytics to understand the market's history and what went wrong with a specific investment (Ganapathy, 2015). The cryptocurrency price emulator software provides crypto users with a view of the entire picture, allowing pre-informed decisions in the cryptocurrency market. Here are some specific ways the cryptocurrency Artificial intelligence Price Emulator can help investors:

It helps investors in choosing the right cryptocurrency investment. Generating income is the main goal of every investor. Cryptocurrency investors need to choose the right crypto investment that will result in profit and not loss. The Emulator AI can help identify the right investment using its price predicting ability. Investors who want to maximize profit by choosing the right investment would find the crypto Emulator AI very reliable for predictive analytics of the cryptocurrency market. It will identify the best crypto investment that will lead to high returns.

It improves the decision-making speed. Finding the right cryptocurrency investment using the manual and normal method involves lots of research. It could take a long while also. The cryptocurrency price Emulator can enable an investor to find the right crypto investment without much personal research and in a shorter time.

Minimizes investment risk. Every investment has some level of risk. The volatility of the cryptocurrency market makes it even riskier to invest. The crypto price emulator reduces investment risk by providing accurate information based on cryptocurrency data gathered. This information can help avoid the harsh effect of the very unstable cryptocurrency market.

Makes cryptocurrency investment finding easier. Crypto price emulator software can remove the complexities associated with cryptocurrency trading and makes it simple to use. Anyone can invest in crypto using the price emulator regardless of their knowledge or background in the cryptocurrency market.

Every Tom, Dick, and Harry may use the emulator to find profitable crypto investment opportunities and even compete with professionals. 


\section{CONCLUSION}

Technology has become part of human life and plays an essential role in our day-to-day activities. Current trends in technological advancement have brought a revolution to so many aspects of human activities, including finance, stock market trade, and investment. Cryptocurrency is one of the many technological advancements which is making massive waves currently. Trading in it has not been easy. To match cryptocurrency market volatility and sharp changes in the market, there is a need for Artificial Intelligence that would make accessing the market more accessible. The machine learns and solves problems copying human Intelligence. The cryptocurrency Artificial intelligence price emulator applies the machine learning concept in data processing and the study's the data to learn the market pattern of the cryptocurrency. This data are collected from cryptorelated news, headlines, social media contents like tweets and comments, cryptocurrency chart history, and then analyzed to reach a result on the possible future. This is an essential improvement in cryptocurrency technology. It would help potential investors, amateur investors, and even professional investors trade in the cryptocurrency market with more conviction and confidence. Many observers and analysts believe that cryptocurrency would significantly impact the financial world in the future. It would advance and become more accepted. The cryptocurrency Artificial intelligence price emulator remains an all-important and must-have tool for cryptocurrency traders.

\section{REFERENCES}

Ganapathy, A. (2015). AI Fitness Checks, Maintenance and Monitoring on Systems Managing Content \& Data: A Study on CMS World. Malaysian Journal of Medical and Biological Research, 2(2), 113-118. https://doi.org/10.18034/mjmbr.v2i2.553

Ganapathy, A. (2016). Speech Emotion Recognition Using Deep Learning Techniques. ABC Journal of Advanced Research, 5(2), 113-122. https://doi.org/10.18034/abcjar.v5i2.550

Narayana, S. L., Suneetha Devi J., Bhargav Reddy I., Harish Paruchuri. (2012). Optimizing Voice Recognition using Various Techniques. CiiT International Journal of Digital Signal Processing, 4(4), $135-141$

Neogy, T. K., \& Paruchuri, H. (2014). Machine Learning as a New Search Engine Interface: An Overview. Engineering International, 2(2), 103-112. https://doi.org/10.18034/ei.v2i2.539

Paruchuri, H. (2015). Application of Artificial Neural Network to ANPR: An Overview. ABC Journal of Advanced Research, 4(2), 143-152. https://doi.org/10.18034/abcjar.v4i2.549

Paruchuri, H. (2017). Credit Card Fraud Detection using Machine Learning: A Systematic Literature Review. ABC Journal of Advanced Research, 6(2), 113-120. https://doi.org/10.18034/abcjar.v6i2.547

Ujwala, D., Ram Kiran, D. S., Jyothi, B., Fathima, S. S., Paruchuri, H., Koushik, Y. M. S. R. (2012). A Parametric Study on Impedance Matching of A CPW Fed T-shaped UWB Antenna. International Journal of Soft Computing and Engineering, 2(2), 433-436.

Vadlamudi, S. (2015). Enabling Trustworthiness in Artificial Intelligence - A Detailed Discussion. Engineering International, 3(2), 105-114. https://doi.org/10.18034/ei.v3i2.519

Vadlamudi, S. (2016). What Impact does Internet of Things have on Project Management in Project based Firms?. Asian Business Review, 6(3), 179-186. https://doi.org/10.18034/abr.v6i3.520

Vadlamudi, S. (2017). Stock Market Prediction using Machine Learning: A Systematic Literature Review. American Journal of Trade and Policy, 4(3), 123-128. https://doi.org/10.18034/ajtp.v4i3.521

$$
--0-
$$

